\title{
Learning to Reach after Learning to Look: a Study of Autonomy in Learning Sensorimotor Transformations.
}

\author{
Claudia Rudolph, Tobias Storck, and Yulia Sandamirskaya \\ Insitute für Neuroinformatik (INI), Ruhr-Univeristät Bochum \\ Universitätsstr. 150, 44780 Bochum, Germany \\ Email: yulia.sandamirskaya@rub.de
}

\begin{abstract}
This paper presents a neurally-inspired architecture for learning to reach toward visually-perceived targets. The whole behavioural loop from object perception to motor control is realised in the architecture of interconnected Dynamic Neural Fields. The sensory-motor mappings, involved in generation of saccadic gaze shifts and reaching arm movements, adapt in the system autonomously along with the generated behaviour. A network of neural-dynamic nodes organises activation and deactivation of the behavioural modules of the architecture in time, leading to an autonomous process model of development of looking and reaching. The architecture was implemented and validated in a simulated robotic agent.
\end{abstract}

\section{INTRODUCTION}

The ability to reach for objects in our immediate environment appears to be so natural and effortless that the complexity of the involved neuronal processes may be easily underestimated. However, this complexity is revealed in developmental studies, which establish that it takes human infants many weeks of intensive (and highly motivated) practice to develop the ability to direct their hands toward interesting objects [1]. The problem of generating a motor plan that directs the hand to a visually perceived object obviously requires learning the sensorimotor transformation between the the visual and the arm systems [2]. This process involves several stages - first, the visual system learns to transform the retinotopic positions of targets into body-centred positions, as revealed, e.g., in developmental studies of double-step saccades [3]. And only later the infant learns to reach [4], i.e. to align the body-centred target representation, generated by the looking system, with the reference frame of the motor system, in which arm movements are planned. All these sensory-motor transformations have not only to be learned initially, but have to be updated constantly as muscles fatigue or the body grows to enable precise looking and reaching movements without visually-driven control [5].

If these complexities were not enough, let us look at the process of generating a reaching movement in an embodied, behaving system. First, a target object has to be selected on the retina, involving top-down and bottom-up attentional processes. Second, the representation of this target's location has to be stabilised (a) against sensory noise and temporal decay of retinal activation at this position and (b) against saccades that might intervene between the initial fixation and reaching onset. For the second stabilisation, (b), the position representation has either to be transformed into body-centred reference frame or the retinotopic representation has to be updated, taking the intervening eye movement into account [6]. Finally, the target representation in a body-centred reference frame, aligned with the motor system of the arm, has to be combined with the current position of the hand to generate the motor command for the hand. This command has to be transformed into joint movements and muscle activation, controlled until the end-point of the movement is reached [7].

Recently, we have introduced a neural-dynamic architecture, based on the Dynamic Field Theory [8], that introduces learning and adaptation of the sensorimotor transformations in an architecture, capable to autonomously generate saccadic eye movements from visual input. We have demonstrated how the involved sensorimotor mapping between the retinal positions of the selected objects and the motor commands, needed to saccade toward these objects may be initially learned and constantly updated [9], [10], [11]. We have implemented the architecture to control an autonomous humanoid robot to show that the model indeed may generate behaviour and learn autonomously in a real-world setting [12].

Here, we extend the neural-dynamic architecture to enable reaching movements, directed at visually perceived objects. In our model for reaching, the gaze-direction independent (bodycentred) representation of the target location is generated by integrating the saccadic motor signal and summing it with the memory of the gaze direction before the saccade. The resulting representation corresponds to the motor state of the eye, in which the eye would be after a precise saccade. In the reaching part of the architecture, we use this gaze-based representation to generate a body-centred target representation, which may control movements of the arm. The integrating factor, required to generate the gaze-based representation, may be learned in order to make a correct prediction of the motor state of the eye after the saccade [12]. The mapping between the gaze-based representation and the proprioceptionbased representation of hand positions is learned subsequently, leading to a developmental cascade: from the ability to look, to the ability to make double-step and memory saccades, and finally the ability to reach for targets.

We implement our extended model on a simulated robot to validate its functioning in an embodied setting and to demonstrate that the system may be used to autonomously calibrate the camera-arm system of a robotic agent. Typically, such calibration is achieved in a manual calibration procedure [13]. Several architectures were developed, which allow to learn 
the proper mapping between visual and arm systems [14], [15], [16]. These architectures use biological inspiration, in particular observation of how human infants learn to coordinate their gaze and arm movements over many trials [17].

In our work, we emphasise the autonomy of learning the sensory-motor transformations. In biological systems, the behaviour and learning are interwoven naturally, the system has to take decisions about when and what to learn based on its own sensory information. The increased autonomy compared to other state-of-the-art systems, in which the sensorimotor transformations are learned on robots in a similar way (e.g., [18], [19]), is realised by a system for behavioural organisation of human and robotic behaviour based on neural dynamics, which we have developed recently [20]. This system activates and deactivates actions - such as attentional shifts, looking movements, and arm movements - and activates learning when appropriate (e.g. when the representations on both sides of a mapping are in correct states, or when an expectation mismatch is detected after an action). We implemented the model in a physically simulated robot CAREN, which consists of a pantilt camera system, a KUKA lightweight robotic arm, and a SCHUNCK hand. The respective hardware setup is available in our lab and will be used to validate and refine the learned mappings in subsequent work.

\section{METHODS}

\section{A. Dynamic Neural Fields}

In our work, we use Dynamic Neural Fields [21], [22], [23] as the mathematical framework for development of the cognitive architecture. From computational perspective, DNFs are attractor dynamics, which stabilise the neural states against noise, fluctuations, and other states, competing for activation. These continuous in time and in space dynamics allow to couple the controlling architecture to real physical sensors and motors, while providing an interface to discrete, or symbolic, cognitive representations [8]. Using DNFs, the whole robotic architecture, including the perceptual and memory systems, behaviour organisation (or planning), learning, and motor control, is formulated as a single (but modular) dynamical system, leading to natural and seamless integration of different components.

The dynamics of a DNF is described by an integrodifferential equation, Eq. (1):

$$
\begin{array}{r}
\tau \dot{u}(x, t)=-u(x, t)+h+\int f\left(u\left(x^{\prime}, t\right)\right) \omega\left(\left|x^{\prime}-x\right|\right) d x^{\prime}+ \\
+I(x, t) .
\end{array}
$$

Here, $u(x, t)$ is the activation of a dynamic neural field (DNF) at time $t ; x$ is one or several behavioural parameters (e.g., colour, location on the retina, postural state, or velocity), over which the DNF is spanned; $\tau$ is the relaxation time-constant of the dynamics; $h$ is the negative resting level that defines the activation threshold of the field; $f(\cdot)$ is the sigmoidal nonlinearity shaping the output of the neural field; the lateral connections in the DNF are shaped by a Mexican hat lateral interaction kernel, $\omega\left(\left|x^{\prime}-x\right|\right)$, with a short-range excitation and a long-range inhibition parts; $I(x, t)$ is the sum of the external inputs to the DNF.
Lateral interactions of the DNFs bring about the existence and stability of a localised-peak solution that is the computational basis for modelling elementary cognitive processes of categorisation, detection, selection, and memory in the DNF framework [8].

Learning in a DNF architecture amounts to an adaptation process in a coupling function, described by the differential equation Eq. (2), [23]:

$$
\begin{aligned}
\tau_{l} \dot{W}(x, y, t)= & c_{\text {learn }}(t)(f(u(x, t)) \times f(v(y, t))) \\
& (-W(x, y, t)+f(u(x, t)) \times f(v(y, t))) .
\end{aligned}
$$

Here, $W(x, y, t)$ expresses the strength of the coupling between two DNFs, e.g., $u(x, t)=W(x, y, t) \cdot v(y, t)$. This coupling reaches an attractor state with a time-constant $\tau_{l}$. The attractor equals the Kronneker product of the outputs of the two fields. The coupling function is only updated when the external learning signal, $c_{\text {learn }}(t)$, is positive and only where the Kronneker product of the outputs of the two coupled DNFs is positive. This learning mechanism expresses an externallydriven (by a system for behavioural organisation or external rewards) associative learning, that increases coupling strength between locations in two DNFs that are activated simultaneously as long as an external signal marks a learning window. Such learning rule is a neural-dynamic equivalent of a neuronal Hebbian learning rule according to which the connections are strengthened between two neuronal populations that are active at the same time ("Fire together, wire together").

The two equations, Eq. (1) and Eq. (2), are used to build the adaptive neural dynamic architecture for generation of looking and reaching, presented next.

\section{THE NEURAL-DYNAMIC ARCHITECTURE}

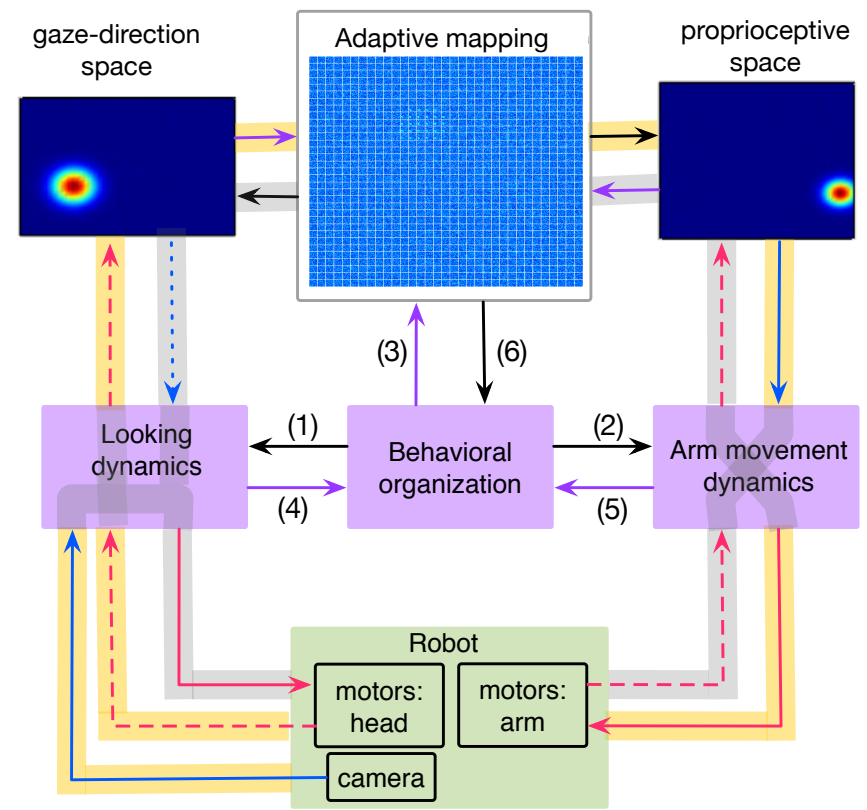

Fig. 1: The dynamical modules of the DNF architecture for learning sensorimotor transformations for looking and reaching. 
The neural-dynamic architecture developed in this work is presented in Fig. 1. The robot (shown by a green box on the bottom of the figure) consists of a camera, mounted on a camera head motor unit, and a robotic arm. The camera drives the looking system (left on the figure), which generates saccadic (i.e. ballistic) camera movements toward objects (here, the salient portions of the visual input). This adaptive looking system has been introduced recently [11], [12], [10] and is described briefly in this paper for completeness in Section III-A. The looking module generates motor commands that drive the motors of the camera head (the pink solid line in the figure), and at the same time creates a representation of the selected object in the gaze-direction space, i.e. in terms of the motor state of the camera head when the robot directs its gaze at the object (top left in the figure). This representation may be generated in the looking module even if the looking movement is not performed by a predictive mapping that generates from the retinotopic (i.e., image-based) location of the target combined with the current gaze-direction - a representation of the gaze direction when the object is centred in the camera image (see predicted gaze-direction DNF on Fig. 2 below).

On the right hand-side of Fig. 1, the arm movement system is depicted, described in Section III-B. The arm movement system receives input from the arm motors about the current position of the end-effector and drives the arm toward the desired end-effector position using standard inverse kinematics and attractor dynamics for movement generation [24]. The desired position of the end-effector coincides with the position of the target object in the proprioceptive space, i.e. the space, in which the end-effector positions resume based on the forward kinematics of the robot (top right in Fig. 1). The mapping between the gaze-direction space and the proprioceptive space allows to generate motor targets based on the visual input. How this mapping is learned autonomously is described in Section III-C.

Next, we describe the individual modules of the architecture in as much detail as space allows us.

\section{A. Looking}

Fig. 2 shows the portion of the neural-dynamic architecture, which generates adaptive gaze shifts (the content of the "Looking dynamics" block in Fig. 1). In this system, the camera provides input to the perceptual Dynamic Neural Field (DNF) - a three-dimensional DNF, defined over the dimensions of the camera image $(x, y)$ (only these two dimensions are shown in the figure), and colour (hue value of the image pixels). The strength of the camera input is the luminance of the image pixels here, although more complex saliency calculations are compatible with this representation [25]. The perceptual DNF builds localised activity peaks over the most salient regions in the retinal (image-based) space.

The target $D N F$ receives the positive activation of the perceptual DNF as input and selects one of the salient regions as the target of the upcoming gaze-shift (saccade) and stabilises the activity peak over this location, transitioning in a selfsustained state. The activity peak in the target field triggers the saccadic burst generator - a number of interconnected dynamic nodes (zero-dimensional DNFs), which generate an oscillation of activation that defines the velocity profile of the upcoming gaze-shift. This velocity profile is multiplied with a gain factor before it is directed to the two motors of the robot (controlling the pan and tilt of the camera head).

The value of the gain factor depends on the location of the target in the image space (larger gains for targets further away from the central part of the image, 'the fovea') and the position of the camera head before the gaze shift. Thus, the gain factors form a map of adaptive gains, which receives inputs from the target $D N F$ and the motor memory $D N F$. The motor memory DNF keeps - as a sustained activation peak the position of the camera head motors before the gaze shift. These two inputs, on the one hand, define the region in the gain map, which is read out during saccade generation to scale the oscillation of the saccadic burst generator. On the other hand, these two inputs define the region of the gain map, in which adaptation takes place after an erroneous saccade: i.e. a saccade that did not bring the target object into the centre of visual field, as detected in the direction of error estimation module. After each gaze shift, the gain maps may be adapted if visual errors are detected and ultimately form a mapping between the image-based space and the motor space of gazeshift commands.

The scaled motor signal is not only sent to the robot's motors, but is also integrated internally and summed with the output of the motor memory DNF, leading to a prediction of the gaze, which the robot would have after the respective saccadic gaze shift. This prediction is a representation of the target object in gaze-based coordinates (one of the body-centred coordinates), which may be used to guide other movements, e.g. the arm movement toward the visually perceived object.

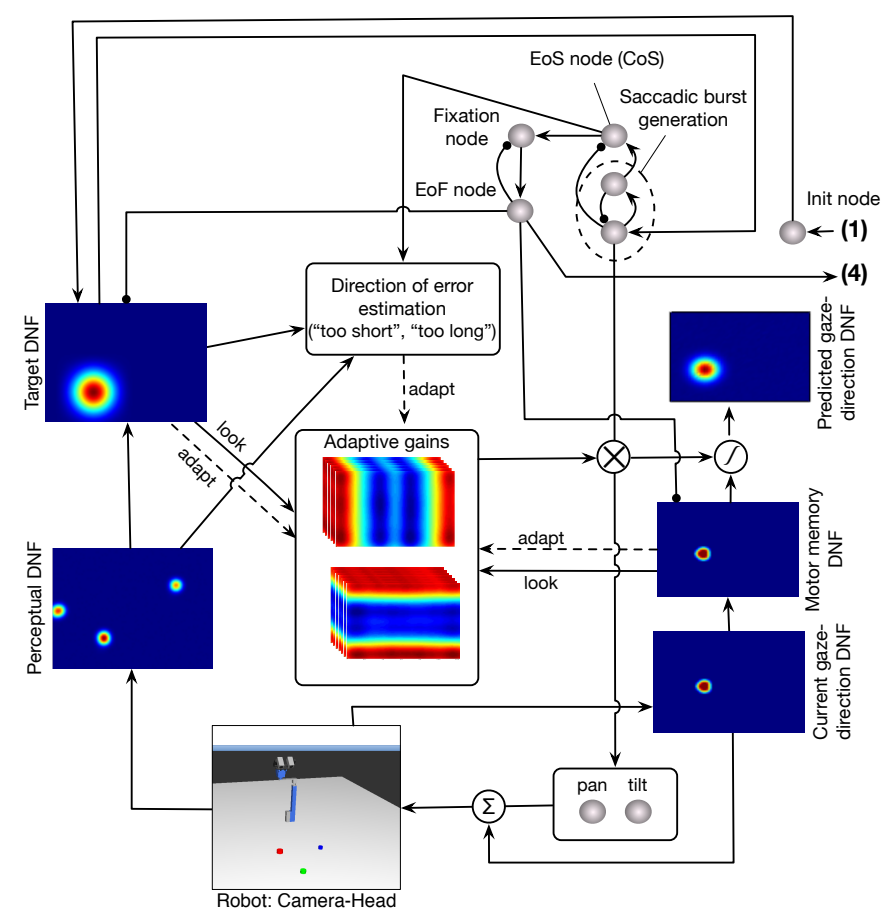

Fig. 2: Architecture for learning to look. 


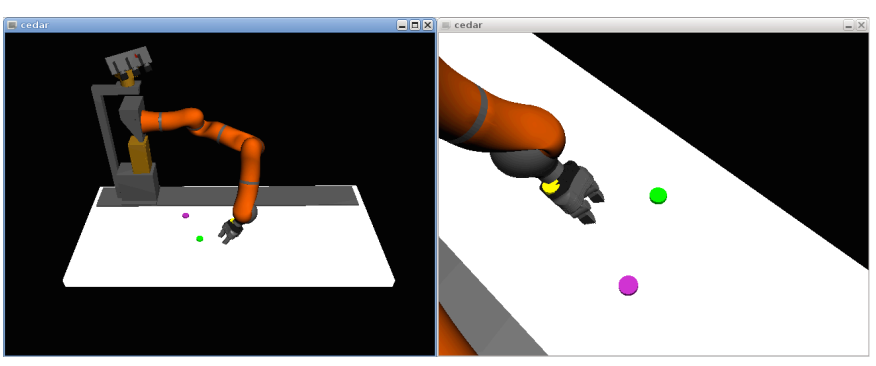

Fig. 3: The simulated robot.

\section{B. Arm movement}

The robotic arm used in our simulated experiments is an eight degrees of freedom KUKA lightweight arm, shown in Fig. 3. The arm movement may be controlled by providing the target position for the end-effector (the hand) in robot-centred coordinates. To calculate the joint-velocities from the target and the starting end-effector positions, the attractor dynamics approach is used, in which the attractor dynamics is defined for the direction of the end-effector movement, Eq. (3):

$$
\tau \dot{\phi}(t)=F_{\text {tar }}\left(\phi, \phi_{\text {tar }}, t\right)+F_{o b s}\left(\phi, \phi_{o b s}, r_{o b s}, t\right) .
$$

Here, $\phi$ is the direction of the end-effector velocity vector relative to an arbitrary reference direction; $\phi_{t a r}$ is the direction of the line, connecting the current position of the end-effector and the target; $\phi_{o b s}, r_{o b s}$ are the direction and distance toward eventual obstacles; $F_{t a r}$ and $F_{o b s}$ are functions that shape the attractors and repellers of the dynamics, respectively (see [26], [24]).

This dynamics drives the end-effector to the target by setting an attractor for the heading direction of the end-effector movement on the direction toward the target (deviating from this direction if obstacles are encountered on the way). In each iteration of the dynamics, the velocity of the end-effector, $v=\left(\phi, v_{t r}\right)\left(v_{t r}\right.$ is the translational component that depends on the distance from the target and obstacles), is translated into joint-velocities using standard inverse kinematics techniques, involving a pseudo-inverse for the redundant manipulator [27].

Thus, the only missing component to perform the arm movement now is the position of the target object in robotcentred ("proprioceptive") coordinates. The mapping between this proprioceptive representation of the target objects and the gaze-centered representation obtained in the looking system may be learned as described next.

\section{Adaptive mapping}

The mapping between the gaze-direction space and the endeffector position space is a four dimensional weights function (implemented as a matrix), which is updated according to Eq. (2), where $x=\left(x_{\text {hand }}, y_{\text {hand }}\right)$ are coordinates of the hand in the plane, parallel to the table, and $y=($ pan, tilt $)$ are the pan and tilt motor components of the robot's gaze. The third component of the hand position - its height over the table is considered to be constant here.

The external learning signal $c_{\text {learn }}(t)=v_{C o S}(t)$ is the output of the condition-of-satisfaction $(C o S)$ node, which signals that the gaze-direction of the robot is aligned with the position of the hand (the robot "looks" at its hand) (see Section III-D). If this $\mathrm{CoS}$ node is active, the weight matrix is updated.

The update happens in the region in the matrix, defined by the Kronneker product of the output of the gaze-direction DNF $u_{\text {gaze }}($ pan, tilt) and the proprioceptive target position DNF $u_{\text {hand }}\left(x_{\text {hand }}, y_{\text {hand }}\right)$ (the gaze-direction space and the proprioceptive space DNFs in Fig. 1 respectively). In this region, the weights approach the output of the Kronneker product, which is positive in the region of the 4D space, where the activity peaks in the two two-dimensional fields overlap.

\section{Behavioral organisation of looking, reaching, and learning}

In order to organisae activation and deactivation of looking, reaching, and learning dynamics at appropriate times, a framework for behavioural organisation with DNFs, developed recently, was used [20].

The two neural-dynamic networks for behavioural organisation are shown in Fig. 4: (a) for generation of reaching movements toward visually perceived targets and (b) for learning the mapping between the gaze-based and proprioceptive reference frames. At their core, the two architectures differ in one node only - the precondition node - which defines whether the camera head is moved first to direct the robot's gaze to an object and the arm is moved then to this object (when reaching action to a visually perceived target is performed), or whether the hand is moved first to an internally generated proprioceptive position and the camera head is moved then to look at the hand (when learning the reference frames transformation). On the periphery, the visual parameter that biases the target object selection in the perceptual DNF of the looking system (Fig. 2) is changed between the two regimes: the target object colour is selected for reaching movement generation and the colour of the robotic hand is selected as the target colour for learning.

In the figure, the red nodes represent intentions of the involved actions [20], and the blue nodes - the respective conditions of satisfactions $(\mathrm{CoS})$, which signal, that the respective action is finished.

For network in Fig. 4a, first, the "Reach" intention node is activated and activates the "move head" and "move hand" intentions, as well as the precondition node between them, which inhibits the "move hand" intention node. The respective $\mathrm{CoS}$ nodes are preactivated, but are only activated when the looking system provides feedback about a completed gaze shift (input (4) from the end-of-fixation (EoF) node of the looking system, see Fig. 2) and when the arm moving system provides feedback about the completed motor action (input (5)). When both actions are complete, the respective CoS nodes are activated and jointly activate the joint $\operatorname{CoS}$ node (centre bottom of Fig. 4a). This CoS node inhibits the "Reach" intention node and, consequently, looses its own activation. The "Reach" intention may be activated again now.

For network in Fig. 4b, first, the "Learn" intention node is activated (equivalent to the "Reach" node above), which activates the "move hand" and "move head" intention nodes and the precondition node, which, this time, inhibits the "move head" intention, ensuring that the hand movement is accomplished first, based on a proprioceptively defined target 


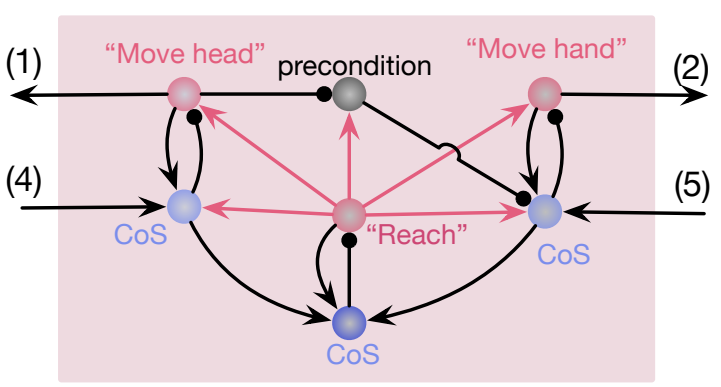

(a) Action generation.

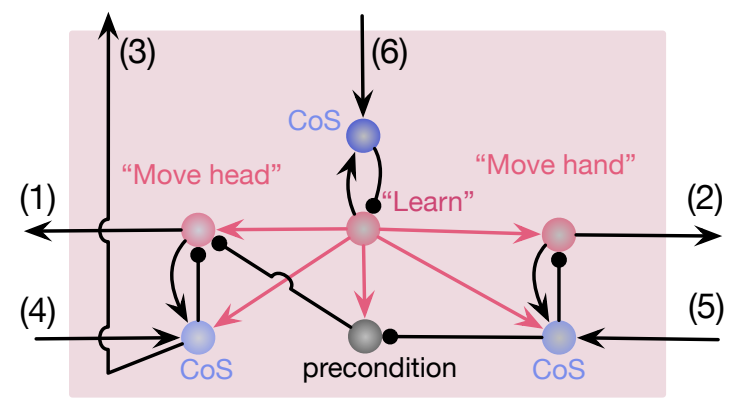

(b) Learning.

Fig. 4: Neural-dynamic networks for behavioural organisation.

location. When the head movement is completed too and the robot "looks" at its hand, learning process is activated through the output (3) $\left(v_{C o S}(t)\right.$ in Section III-C). When learning has saturated, the input (6) activates the $\mathrm{CoS}$ that inhibits the "Learn" intention node. Loosing input from the "Learn" intention node, the CoS's activation ceases, the intention node is released from inhibition, and the new learning act may begin.

Numbers on the input and output arrows in Fig. 4 correspond to numbers in Fig. 1: (1) is the input to the init node of the looking dynamics, which initiates a gaze-shift action, (4) is the output of the end-of-fixation (EoF) node of the looking dynamics that signals that the gaze-shift is finished, (2) is the input that initiates arm movement dynamics, (5) is the output of the arm movement system, which signals that the hand has reached the target, (3) activaties adaptation of the coupling function, (6) signals that the adaptation iteration is complete. Lines with circles in Fig. 4 mark inhibitory connections in the network.

The networks in Fig. 4 allow the system to perform reaching movements toward visually perceived targets and update the mapping in order to generate precise arm movements autonomously. Switching between these two regimes is done manually here, but could be organised in a hierarchical system for behavioral organisation [28].

\section{RESUlTS}

The model, described above was implemented in a simulated robot CAREN, Fig. 3. The looking part of the model controlled the robot's head and received input from the robotic camera, the arm movement dynamics moved the robotic arm, as described above. Next, we present some results of the simulated experiments, which demonstrate how the mappings, involved in looking and reaching, were autonomously learned.
The four-dimensional weight matrix, learned for our simulated robot, is shown in Fig. 5. The tiles in the figure span the proprioceptive motor space (positions of the hand over the table in body-centred coordinates used to move the arm). The tiles are aligned along visual gaze-direction coordinates (pan and tilt of the camera head). The red regions in the map are regions with positive values, which connect the locations in the gaze-based body-centred space with the locations in the proprioceptive body-centred space. Blue regions represent small values that are close to zero. The map is updated in the regions that were visited by the gaze and the hand during the learning process. Some degree of generalisation is achieved, because the learning regions have a finite width, defined by the width of the activity peaks in the gaze-based and the proprioceptive DNFs (and are not single points).

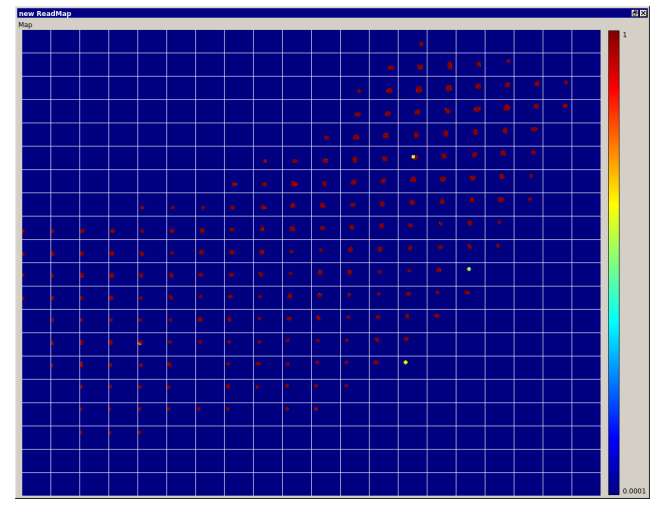

Fig. 5: Map.

Apart from this "local" generalisation, the quality of the learned map depends on the learning process and the environment, in which the autonomous learning unfolds. If learning is performed in a stationary environment, mapping can be learned only for the positions, at which the objects are present.

Fig. 6 shows values of the reaching errors depending on the position of the target on the table after the map was learned. During learning, objects were placed in the simulated environment on the table on a grid with $10 \mathrm{~cm} \times 10 \mathrm{~cm}$ cells. The error stays below $5 \mathrm{~cm}$ in all tested positions, even those between the grid nodes.

Fig. 7 demonstrates the autonomy of the process of generation of reaching and looking movements during learning. In particular, the sigmoided activation of the intention nodes of the system for behavioural organisation in a learning experiment (Fig. 4b) are shown. First, the look and learn node is activated, followed shortly by the move hand node. When this arm movement to an internally generated motor target ${ }^{1}$ is completed, the respective $\mathrm{CoS}$ node is activated and inhibits the move hand intention, as well as the precondition node, which was inhibiting the look at hand intention (Fig. 4b). The look at hand intention node is activated then and drives the camera head to direct the robot's gaze to the robot's hand. When this movement is completed (as detected by the respective CoS node, which receives inputs from the saccadic burst generator

\footnotetext{
1"goal babbling" could be performed here, but a systematic presentation of motor goals from a grid that sampled the motor space over the table, was used here instead
} 


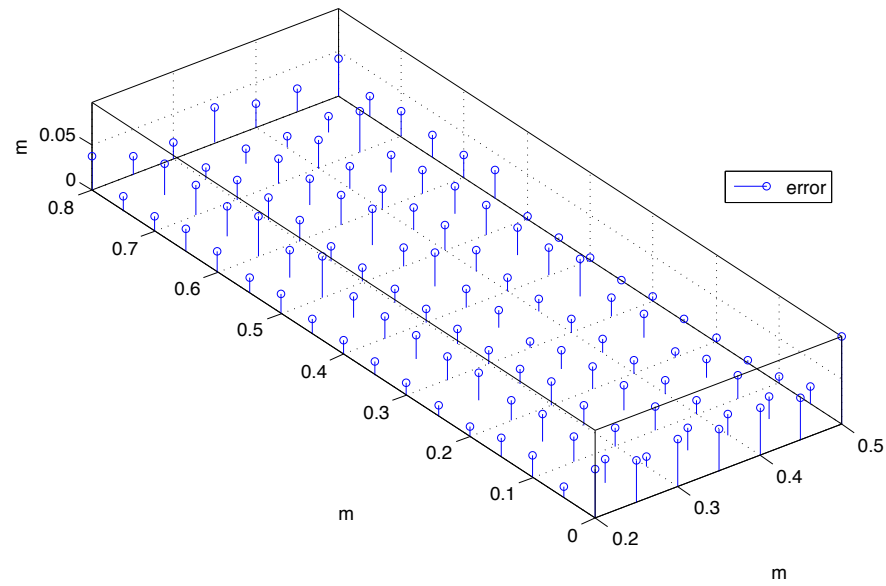

Fig. 6: Reaching errors depending on the position of the object on the table.

of the looking system), the look at hand intention is inhibited and the learning process is activated, updating the coupling function according to Eq. 2. The "look and learn" intention is deactivated when the learning process saturates, which results in a short learning window between the drop of the red curve and the drop of the green curve in Fig. 7.

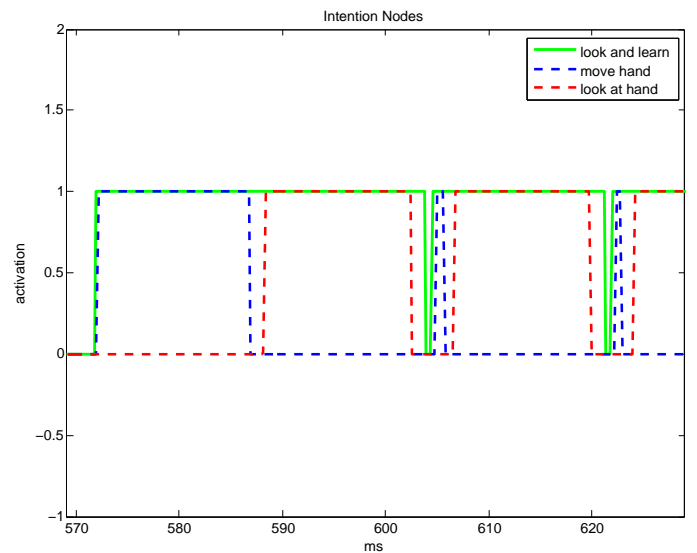

Fig. 7: Time course of activation of the intention nodes of the network for behavioural organisation during three successive looking and reaching movements during map learning.

Note, that during the second activation of the "look and learn" intention, the move hand intention (blue line in Fig. 7) is only activated briefly, since the robot performs only a short movement to the next position on the exploration grid (remember, the motor goals are presented to the robot in an ordered way here). When this short movement is completed, the $\mathrm{CoS}$ of the move hand action is activated and triggeres the next behaviour. The system can handle such different durations of actions since the behaviour organisation network ensures that every module of the neural-dynamic architecture is activated and deactivated as appropriate.

Fig. 8 shows the precision of the learned movements. Blue circles show the position of the robotic hand at the end of each movement after learning the map, red crosses show the projection onto the table of the gaze direction of the robot (the point on the table which was in the centre of the camera image at the end of the respective gaze shift). The black dot represents the target object. The scales on the axes are in $\mathrm{cm}$. The small rest variability comes from the distributed representation of the mapping, updates of the mapping that co-occur with the behaviour, and imprecisions in localisation of the objects in the perceptual and target DNFs. This variability is comparable to the variability of human reaching and looking movements [29], [30].

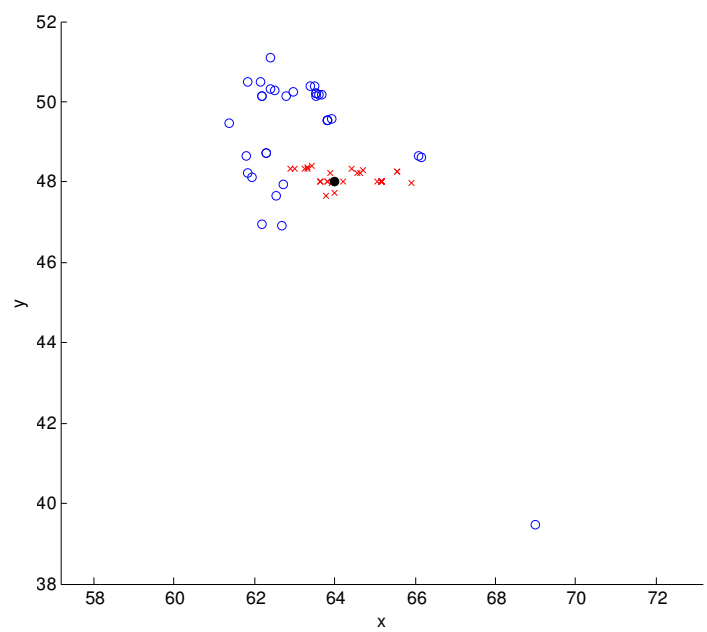

Fig. 8: Errors of looking and reaching.

Fig. 9a shows looking and reaching movements, performed autonomously toward three targets, present on the tabletop. Each target was accessed three times in a succession. The targets were selected autonomously, as well as the movements were performed and their completion was detected autonomously. Fig. 9b shows looking and reaching movements towards nine different targets, located on an arc in front of the robot. Again, the targets were selected autonomously and the looking and reaching movements initiated and terminated autonomously using DNF dynamics and networks for behavioural organisation.

\section{CONCLUSION}

This paper presents a neural-dynamic architecture for autonomous generation of reaching movements toward visually perceived targets. The model comprises the whole behavioural loop from selection and stabilisation of the visual target, generation of camera movement and adaptation of the involved sensory-motor mapping, learning of transformation between the visual gaze-based space and the proprioceptive handspace, and generation of the goal-directed arm movement. Autonomous learning of the transformation, which maps the gaze-based representation of the target onto the proprioceptive representation of the target, which may be used to drive arm movements, is the focus of this paper and was demonstrated in a number of simulated robotic experiments.

\section{REFERENCES}

[1] D. Corbetta, S. L. Thurman, R. F. Wiener, Y. Guan, and J. L. Williams, "Mapping the feel of the arm with the sight of the object: on the embodied origins of infant reaching." Frontiers in psychology, vol. 5, no. June, p. 576, Jan. 2014. 


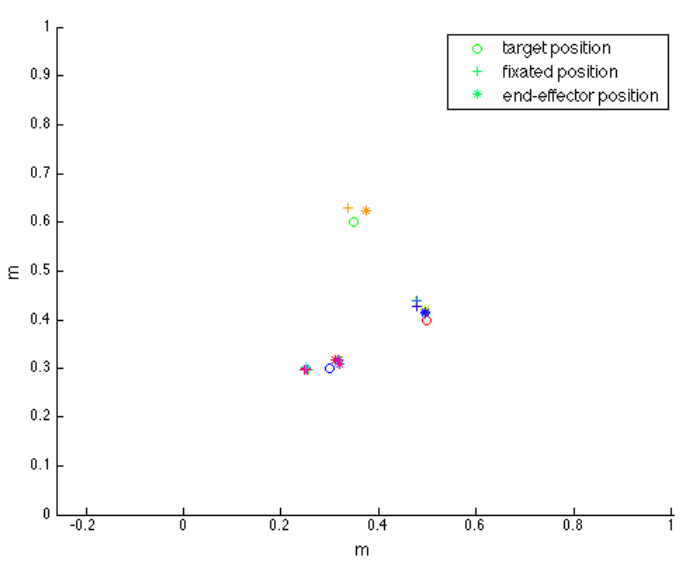

(a) Moving in a circle.

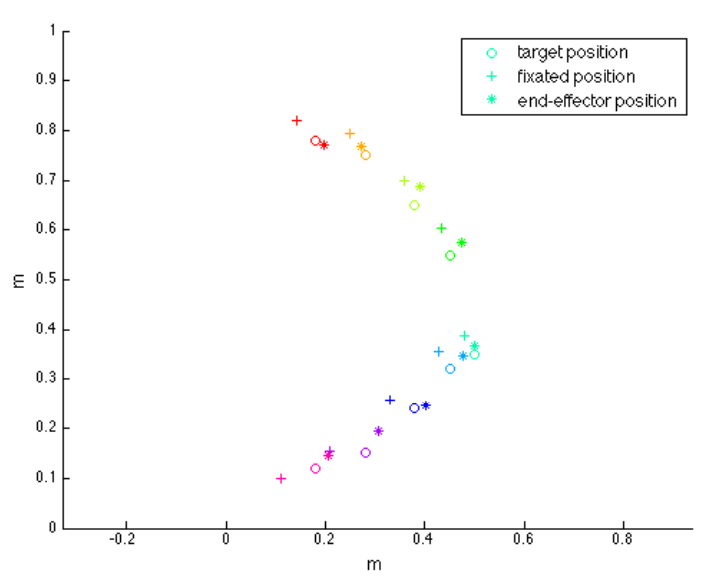

(b) Moving on an arc.

Fig. 9: Autonomous sequence of looking and reaching movements.

[2] R. Shadmehr and S. Wise, "Motor learning and memory for reaching and pointing," The new cognitive neurosciences, pp. 511-524, 2003.

[3] R. O. Gilmore and M. H. Johnson, "Body-centered representations for visually-guided action emerge during early infancy," Cognition, vol. 65 , pp. 1-9, 1997.

[4] C. von Hofsten, "Structuring of Early reaching Movemens: A longitudinl Study," Journal of motor behavior, 1991

[5] D. Pelisson and N. Alahyane, "Sensorimotor adaptation of saccadic eye movements," Neuroscience \& Biobehavioral Reviews, vol. 34, no. 8, pp. 1103-1120, 2010.

[6] R. A. Andersen, L. H. Snyder, D. C. Bradley, and J. Xing, "Multimodal representation of space in the posterior parietal cortex and its use in planning movements," Annual Reviews of Neuroscience, vol. 20, pp. 303-330, 1997.

[7] P. Gaudiano and S. Grossberg, "Adaptive vector integration to endpoint: Self-organizing neural circuits for control of planned movement trajectories," Human Movement Science, 1992.

[8] Y. Sandamirskaya, S. K. U. Zibner, S. Schneegans, and G. Schöner, "Using Dynamic Field Theory to extend the embodiment stance toward higher cognition," New Ideas in Psychology, vol. 31, no. 3, pp. 322-339, 2013.

[9] Y. Sandamirskaya and J. Conradt, "Learning Sensorimotor Transformations with Dynamic Neural Fields," in International Conference on Artificial Neural Networks (ICANN), 2013.

[10] C. Bell, T. Storck, and Y. Sandamirskaya, "Learning to look: a dynamic neural fields architecture for gaze shift generation," in International
Conference for Artificial Neural Networks, ICANN, Hamburg, Germany, 2014.

[11] P. Koprinkova-Hristova, V. Mladenov, and N. K. Kasabov, Eds., Artificial Neural Networks. Springer, 2015, vol. 4, ch. Learning to look and looking to remember: a neural-dynamic embodied model for generation of saccadic gaze shifts and memory formation.

[12] Y. Sandamirskaya and T. Storck, "Neural-dynamic architecture for looking: Shift from visual to motor target representation for memory saccade," in IEEE International Conference on Development and Learning and on Epigenetic Robotics (ICDL EPIROB 2014), 2014.

[13] R. Tsai, "A Versatile Camera Calibration Technique for high-Accuracy 3D Machine Vision Metrology Using Off-the-Shelf TV Cameras and Lenses," Robotics and Automation, IEEE Journal of, vol. 3, no. 4, pp. 323-344, 1987.

[14] R. Saegusa, G. Metta, G. Sandini, and S. Sakka, "Active motor babbling for sensorimotor learning," in Robotics and Biomimetics, 2008. ROBIO 2008. IEEE International Conference on. IEEE, 2009, pp. 794-799.

[15] M. Kuperstein, "Neural model of adaptive hand-eye coordination for single postures," Science, vol. 239, pp. 1308-1311, 1988.

[16] F. Guenther, D. Bullock, D. Greve, and S. Grossberg, "Neural representations for sensory-motor control. $\{\mathrm{III}\}$. $\{\mathrm{L}\}$ earning a bodycentered representation of 3-D target position," Journal of Cognitive Neuroscience, vol. 6, pp. 341-358, 1994.

[17] J. D. Crawford, W. P. Medendorp, and J. J. Marotta, "Spatial transformations for eye-hand coordination." Journal of neurophysiology, vol. 92, no. 1, pp. 10-9, Jul. 2004.

[18] G. Metta, G. Sandini, and J. Konczak, "A developmental approach to visually-guided reaching in artificial systems." Neural networks : the official journal of the International Neural Network Society, vol. 12, no. 10, pp. 1413-1427, Dec. 1999.

[19] G. Guilherme, A. F. R. Araújo, and H. J. Ritter, "Self-organizing feature maps for modeling and control of robotic manipulators," Journal of Intelligent \&amp; Robotic Systems, vol. 36, no. 4, pp. 407-450, 2003.

[20] M. Richter, Y. Sandamirskaya, and G. Schöner, "A robotic architecture for action selection and behavioral organization inspired by human cognition," in IEEE/RSJ International Conference on Intelligent Robots and Systems, IROS, 2012.

[21] S. Amari, "Dynamics of pattern formation in lateral-inhibition type neural fields," Biological Cybernetics, vol. 27, pp. 77-87, 1977.

[22] G. Schöner, Dynamical systems approaches to cognition. Cambridge University Press, 2008.

[23] Y. Sandamirskaya, "Dynamic Neural Fields as a Step Towards Cognitive Neuromorphic Architectures," Frontiers in Neuroscience, vol. 7, p. 276, 2013.

[24] H. Reimann, I. Iossifidis, and G. Schöner, "Autonomous movement generation for manipulators with multiple simultaneous constraints using the attractor dynamics approach," in IEEE International Conference on Robotics and Automation (ICRA), Shanghai, China, 2011, pp. 10504729.

[25] L. Itti and C. Koch, "Computational modeling of visual attention," Nature reviews neuroscience, vol. 2, pp. 1-11, 2001.

[26] E. Bicho and G. Schöner, "Target position estimation, target acquisition, and obstacle avoidance," in Proceedings of the IEEE International Symposium on Industrial Electronics (ISIE'97). IEEE, Piscataway, NJ, 1997, pp. SS13-SS20.

[27] B. Siciliano, "Kinematic control of redundant robot manipulators: A tutorial," Journal of Intelligent and Robotic Systems, vol. 3, no. 3, pp. 201-212, 1990.

[28] Duran B and Y. Sandamirskaya, "Neural Dynamics of Hierarchically Organized Sequences: a Robotic Implementation," in Proceedings of 2012 IEEE-RAS International Conference on Humanoid Robots (Humanoids), 2012.

[29] J. B. J. Smeets and I. T. C. Hooge, "Nature of variability in saccades." Journal of neurophysiology, vol. 90, pp. 12-20, 2003.

[30] J. Messier and J. F. Kalaska, "Comparison of variability of initial kinematics and endpoints of reaching movements," Experimental Brain Research, vol. 125, pp. 139-152, 1999. 